\title{
Newton-Type Iteration for Tikhonov Regularization of Nonlinear Ill-Posed Problems
}

\author{
Santhosh George \\ Department of Mathematical and Computational Sciences, National Institute of Technology, Mangalore, Karnataka 575 025, India
}

Correspondence should be addressed to Santhosh George; nitksanthosh@gmail.com

Received 14 August 2012; Accepted 15 October 2012

Academic Editor: De-Xing Kong

Copyright (C) 2013 Santhosh George. This is an open access article distributed under the Creative Commons Attribution License, which permits unrestricted use, distribution, and reproduction in any medium, provided the original work is properly cited.

Recently in the work of George, 2010, we considered a modified Gauss-Newton method for approximate solution of a nonlinear ill-posed operator equation $F(x)=y$, where $F: D(F) \subseteq X \rightarrow Y$ is a nonlinear operator between the Hilbert spaces $X$ and $Y$. The analysis in George, 2010 was carried out using a majorizing sequence. In this paper, we consider also the modified Gauss-Newton method, but the convergence analysis and the error estimate are obtained by analyzing the odd and even terms of the sequence separately. We use the adaptive method in the work of Pereverzev and Schock, 2005 for choosing the regularization parameter. The optimality of this method is proved under a general source condition. A numerical example of nonlinear integral equation shows the performance of this procedure.

"Dedicated to Prof. Ulrich Tautenhahn"

\section{Introduction}

Inverse problems have been one of the fastest growing research area in applied mathematics in the last decades. It is well known that these problems typically lead to mathematical models that are ill-posed (according to Hadamard's definition [1]) in the sense that it is not possible to provide a unique solution.

In this paper, we consider the task of approximately solving the nonlinear ill-posed equation

$$
F(x)=y .
$$

This equation and the task of solving it make sense only when placed in an appropriate framework. Throughout this paper, we will assume that $F: D(F) \subseteq X \rightarrow Y$ is a nonlinear operator between Hilbert spaces $X$ and $Y$ with inner product and corresponding norm denoted by $\langle\cdot, \cdot\rangle$ and $\|\cdot\|$, respectively, and $y \in Y$. We assume that (1) has a unique solution $\widehat{x}$. For $\delta>0$, let $y^{\delta} \in Y$ be an available data with

$$
\left\|y-y^{\delta}\right\| \leq \delta
$$

Since (1) is ill-posed, regularization methods are used to obtain stable approximate solutions [2,3]. Iterative regularization methods are one such class of regularization methods [4-8].

In [4], Bakushinskii proposed an iterative method, namely, the iteratively regularized Gauss-Newton method, in which the iterations are defined by

$$
\begin{aligned}
x_{k+1}^{\delta}= & x_{k}^{\delta}-\left(\alpha_{k} I+A_{k, \delta}^{*} A_{k, \delta}\right)^{-1} A_{k, \delta}^{*} \\
& \times\left[F\left(x_{k}^{\delta}\right)-y^{\delta}+\alpha_{k}\left(x_{k}^{\delta}-x_{0}\right)\right], \quad x_{0}^{\delta}=x_{0},
\end{aligned}
$$

where $A_{k, \delta}:=F^{\prime}\left(x_{k}^{\delta}\right)$ (here and in the following $F^{\prime}$ denotes the Fréchet derivative of $F)$ and $\left(\alpha_{k}\right)$ is a sequence of real numbers satisfying

$$
\alpha_{k}>0, \quad 1 \leq \frac{\alpha_{k}}{\alpha_{k+1}} \leq \mu_{1}, \quad \lim _{k \rightarrow 0} \alpha_{k}=0
$$

for some constant $\mu_{1}>1$. For convergence analysis, Bakushinskii used the following Hölder-type source condition on the exact solution $\hat{x}$ of (1)

$$
x_{0}-\widehat{x}=A^{*} A w, \quad A:=F^{\prime}(\widehat{x}),
$$


for some $w \in X$. Later Hohage $[9,10]$ and Langer and Hohage [11] considered the iteratively regularized Gauss-Newton method under different source conditions and stopping rules.

In [5], Bakushinskii generalized the procedure in [4] by considering a generalized form of the regularized GaussNewton method in which the iterations are defined by

$$
\begin{aligned}
x_{k+1}^{\delta}= & x_{0}-g_{\alpha_{k}}\left(A_{k, \delta}^{*} A_{k, \delta}\right) A_{k, \delta}^{*} \\
& \times\left[\left(F\left(x_{k}^{\delta}\right)-y^{\delta}\right)-A_{k, \delta}\left(x_{k}^{\delta}-x_{0}\right)\right], \quad x_{0}^{\delta}=x_{0},
\end{aligned}
$$

where $A_{k, \delta}$ and $\left(\alpha_{k}\right)$ are as in (3), and each $g_{\alpha}$ for $\alpha>0$ is a piecewise continuous function. It should be noted that the convergence of (3) was also shown by Bakushinsky and Smirnova in [12]. In [6], Blaschke et al. considered the above generalized procedure under a stopping index $k_{\delta}$ such that

$$
\begin{aligned}
& \left\|F\left(x_{k_{\delta}}^{\delta}\right)-y^{\delta}\right\| \\
& \quad \leq c \delta<\left\|F\left(x_{k}^{\delta}\right)-y^{\delta}\right\|, \quad k=0,1, \ldots, k_{\delta}-1, c>1,
\end{aligned}
$$

and the error estimate is obtained under the following Hölder-type source condition:

$$
x_{0}-\widehat{x}=\left(A^{*} A\right)^{v} w, \quad 0<v \leq 1 .
$$

Recently, Mahale and Nair [8], considered the iteration procedure:

$$
\begin{aligned}
x_{k+1}^{\delta}= & x_{0}-g_{\alpha_{k}}\left(A_{0}^{*} A_{0}\right) A_{0}^{*} \\
& \times\left[\left(F\left(x_{k}^{\delta}\right)-y^{\delta}\right)-A_{0}\left(x_{k}^{\delta}-x_{0}\right)\right], \quad x_{0}^{\delta}=x_{0},
\end{aligned}
$$

where $A_{0}:=F^{\prime}\left(x_{0}\right),\left(\alpha_{k}\right)$ is as in (3), and $g_{\alpha}$ for $\alpha>0$ is a positive real-valued piecewise continuous function defined in $[0, M]$ with $M \geq\left\|A_{0}\right\|^{2}$. In [8], the stopping index $k_{\delta}$ for the iteration is chosen such that

$$
\begin{aligned}
\max & \left\{\left\|F\left(x_{k_{\delta}-1}^{\delta}\right)-y^{\delta}\right\|, \beta_{k_{\delta}}\right\} \\
& \leq \tau \delta<\max \left\{\left\|F\left(x_{k-1}^{\delta}\right)-y^{\delta}\right\|, \beta_{k}\right\}
\end{aligned}
$$

$1 \leq k<k_{\delta}$, where $\tau>0$ is a sufficiently large constant not depending on $\delta$, and

$$
\beta_{k}:=\left\|F\left(x_{k-1}^{\delta}\right)-y^{\delta}+A_{0}\left(x_{k}^{\delta}-x_{k-1}^{\delta}\right)\right\| .
$$

To prove the results in [8], Mahale and Nair considered the following general source condition:

$$
x_{0}-\widehat{x}=\left[\varphi\left(A_{0}^{*} A_{0}\right)\right]^{1 / 2} w,
$$

for some $w \in X$ with $\|w\| \leq \rho, \rho>0$. Here, $\varphi$ : $(0, M] \rightarrow(0, \infty)$ is a continuous, strictly monotonically increasing function satisfying $\lim _{\lambda \rightarrow 0} \varphi(\lambda)=0$ with $M \geq$ $\left\|A_{0}\right\|^{2}$
Note that the source conditions (5) and (8) involves the Fréchet derivative at the exact solution $\widehat{x}$ which is unknown in practice. But the source condition (12) depends on the Fréchet derivative of $F$ at $x_{0}$.

In [7], Kaltenbacher considered the following iteration procedure:

$$
\begin{aligned}
x_{n+1, \alpha}^{\delta}=\operatorname{Proj}_{D(F)}[ & x_{n, \alpha}^{\delta}-\left(A_{n}^{*} A_{n}+\alpha I\right)^{-1} \\
& \left.\times\left(A_{n}^{*}\left(F\left(x_{n, \alpha}^{\delta}\right)-y^{\delta}\right)+\alpha\left(x_{n, \alpha}^{\delta}-x_{0}\right)\right)\right],
\end{aligned}
$$

where $A_{n}:=F^{\prime}\left(x_{n, \alpha}^{\delta}\right), x_{0}=\operatorname{Proj}_{D(F)} x_{0, \alpha}^{\delta}$ and proved that the sequence $\left(x_{n, \alpha}^{\delta}\right)$ converges to the critical point $x_{\alpha}^{\delta}$ of the Tikhonov functional, characterized by $F^{\prime}\left(x_{\alpha}^{\delta}\right)^{*}\left(F\left(x_{\alpha}^{\delta}\right)-y^{\delta}\right)+$ $\alpha\left(x_{\alpha}^{\delta}-x_{0}\right)=0$. In order to obtain an error estimate of $\left\|x_{\alpha}^{\delta}-\widehat{x}\right\|$ in [7], the following two kinds of conditions are used:

(a) $F^{\prime}(\bar{x})=R(\bar{x}, x) F^{\prime}(x),\|R(\bar{x}, x)-I\| \leq C_{R}\|\bar{x}-x\|^{\kappa}$ for $x, \bar{x} \in B_{\rho}\left(x_{0}\right)$,

(b) $F^{\prime}(\bar{x})=F^{\prime}(x) R(\bar{x}, x),\|R(\bar{x}, x)-I\| \leq C_{R}\|\bar{x}-x\|^{\kappa}$ for $x, \bar{x} \in B_{\rho}\left(x_{0}\right), \kappa \in[0,1]$,

with

$$
\left(1+C_{R}(2 \rho)^{\kappa}\right)^{2}\left(\frac{C_{R}}{2}\right)(2 \rho)^{\kappa}<1
$$

for condition (a) and

$$
2 C_{R} \rho<1
$$

for condition (b).

In [13], the author considered a particular case of the method (9), that is,

$$
\begin{aligned}
x_{n+1, \alpha}^{\delta}= & x_{n, \alpha}^{\delta}-\left(A_{0}^{*} A_{0}+\alpha I\right)^{-1} \\
& \times\left[A_{0}^{*}\left(F\left(x_{n, \alpha}^{\delta}\right)-y^{\delta}\right)+\alpha\left(x_{n, \alpha}^{\delta}-x_{0}\right)\right], \\
& x_{0, \alpha}^{\delta}=x_{0},
\end{aligned}
$$

or equivalently,

$$
\begin{aligned}
x_{n+1, \alpha}^{\delta}= & x_{0}-\left(A_{0}^{*} A_{0}+\alpha I\right)^{-1} A_{0}^{*} \\
& \times\left[\left(F\left(x_{n, \alpha}^{\delta}\right)-y^{\delta}\right)-A_{0}\left(x_{n, \alpha}^{\delta}-x_{0}\right)\right], \\
& x_{0, \alpha}^{\delta}=x_{0},
\end{aligned}
$$

for approximately solving (1). Analysis in [13], was carried out using a suitably constructed majorizing sequence, and the stopping rule in [13] was based on this majorizing sequence.

Recall [14, Definition 1.3.11], that a nonnegative increasing sequence $\left(t_{n}\right)$ (i.e., $t_{n+1}-t_{n} \geq 0$ ) is said to be a majorizing sequence of a sequence $\left(x_{n}\right)$ in $X$, if

$$
\left\|x_{n+1}-x_{n}\right\| \leq t_{n+1}-t_{n}, \quad \forall n \geq 0 .
$$

The majorizing sequence in [13], depends on the initial guess $x_{0}$, and the conditions on $x_{0}$ (see, e.g., (3.2) in [13]) 
are restrictive, so the method is not suitable for practical consideration.

In this paper, we consider the sequence (17) and analyze it by considering its even and odd terms separately and obtain the optimal order of the error. The regularization parameter $\alpha$ is chosen according to the balancing principle considered by Pereverzev and Schock in [15].

The organization of this paper is as follows. Proposed method and its convergence analysis are given in Section 2. Error analysis and parameter choice strategy are discussed in Section 3. Section 4 deals with the implementation of the method; a numerical example is given in Section 5, and finally, the paper ends with a conclusion in Section 6.

\section{Convergence of the Method (17)}

Let

$$
\begin{gathered}
y_{n}^{\delta}:=x_{0}-R_{\alpha}^{-1} A_{0}^{*}\left[F\left(x_{n}^{\delta}\right)-y^{\delta}-A_{0}\left(x_{n}^{\delta}-x_{0}\right)\right], \\
x_{n+1}^{\delta}:=x_{0}-R_{\alpha}^{-1} A_{0}^{*}\left[F\left(y_{n}^{\delta}\right)-y^{\delta}-A_{0}\left(y_{n}^{\delta}-x_{0}\right)\right],
\end{gathered}
$$

where $R_{\alpha}:=A_{0}^{*} A_{0}+\alpha I$ and $x_{0}$ is the initial guess.

Remark 1. It can be seen that $y_{n}^{\delta}=x_{2 n-1, \alpha}^{\delta}$ and $x_{n+1}^{\delta}=x_{2 n, \alpha}^{\delta}$, where $x_{n, \alpha}^{\delta}$ is defined as in (17).

Assumption 2. There exists a constant $k_{0}>0$ such that for every $x, u \in D(F)$ and $v \in X$, there exists an element $\Phi(x, u, v) \in X$ satisfying

$$
\begin{gathered}
{\left[F^{\prime}(x)-F^{\prime}(u)\right] v=F^{\prime}(u) \Phi(x, u, v),} \\
\|\Phi(x, u, v)\| \leq k_{0}\|v\|\|x-u\|
\end{gathered}
$$

for all $x, u \in D(F)$ and $v \in X$.

Let

$$
e_{n}^{\delta}:=\left\|y_{n}^{\delta}-x_{n}^{\delta}\right\|, \quad \forall n \geq 0
$$

Hereafter, for convenience, we use the notation $x_{n}, y_{n}$, and $e_{n}$ for $x_{n}^{\delta}, y_{n}^{\delta}$, and $e_{n}^{\delta}$, respectively.

Let $\delta_{0}<\sqrt{\alpha_{0}} / 4 k_{0}, 0<\rho \leq\left(1 / k_{0}\right)\left[\sqrt{(3 / 2)-\left(2 k_{0} \delta_{0} / \sqrt{\alpha_{0}}\right)}\right.$ $-1]$, the parameter $\alpha$ is selected from some finite set

$$
\begin{gathered}
D_{M}:=\left\{\alpha_{i}: 0<\alpha_{0}<\alpha_{1}<\cdots<\alpha_{M}\right\}, \\
\gamma_{\rho}:=\frac{\delta_{0}}{\sqrt{\alpha_{0}}}+\frac{k_{0}}{2} \rho^{2}+\rho .
\end{gathered}
$$

Throughout this paper, we assume that the operator $F$ is Fréchet differentiable at all $x \in D(F)$.

Remark 3. Note that if $\left\|x_{0}-\widehat{x}\right\| \leq \rho$, then by Assumption 2, we have

$$
e_{0} \leq \gamma_{\rho}
$$

This can be seen as follows:

$$
\begin{aligned}
e_{0}= & \left\|y_{0}-x_{0}\right\| \\
= & \left\|R_{\alpha}\left(x_{0}\right)^{-1} A_{0}^{*}\left(y^{\delta}-F\left(x_{0}\right)\right)\right\| \\
= & \| R_{\alpha}\left(x_{0}\right)^{-1} A_{0}^{*}\left(y^{\delta}-y+F(\widehat{x})-F\left(x_{0}\right)\right. \\
& \left.\quad-A_{0}\left(\widehat{x}-x_{0}\right)+A_{0}\left(\widehat{x}-x_{0}\right)\right) \| \\
\leq & \left\|R_{\alpha}\left(x_{0}\right)^{-1} A_{0}^{*}\left(y^{\delta}-y\right)\right\| \\
& +\left\|R_{\alpha}\left(x_{0}\right)^{-1} A_{0}^{*} \int_{0}^{1}\left[F^{\prime}\left(x_{0}+t\left(\widehat{x}-x_{0}\right)\right)-A_{0}\right] d t\left(\widehat{x}-x_{0}\right)\right\| \\
& +\left\|R_{\alpha}\left(x_{0}\right)^{-1} A_{0}^{*} A_{0}\left(\widehat{x}-x_{0}\right)\right\| \\
\leq & \frac{\delta}{\sqrt{\alpha}}+\frac{k_{0}}{2} \rho^{2}+\rho \\
\leq & \frac{\delta_{0}}{\sqrt{\alpha_{0}}}+\frac{k_{0}}{2} \rho^{2}+\rho \\
= & \gamma_{\rho} \leq \frac{1}{4 k_{0}} .
\end{aligned}
$$

Using the inequality (24), we prove the following.

Theorem 4. Let $\left\|x_{0}-\widehat{x}\right\| \leq \rho, q=k_{0} r$, where $r \in((1-$ $\left.\left.\sqrt{1-4 k_{0} \gamma_{\rho}}\right) / 2 k_{0},\left(1+\sqrt{\left(1-4 k_{0} \gamma_{\rho}\right)}\right) / 2 k_{0}\right)$. Let $e_{n}$ be as in (22), and let $x_{n}$ and $y_{n}$ be as in (19) and (20), respectively, with $\delta \in\left[0, \delta_{0}\right)$ and $\alpha \in D_{M}$. Then, we have the following:
(a) $\left\|x_{n}-y_{n-1}\right\| \leq q\left\|y_{n-1}-x_{n-1}\right\|$,
(b) $\left\|y_{n}-x_{n}\right\| \leq q^{2}\left\|y_{n-1}-x_{n-1}\right\|$,
(c) $e_{n} \leq q^{2 n} \gamma_{\rho}$,
(d) $x_{n}, y_{n} \in B_{r}\left(x_{0}\right):=\left\{x \in X:\left\|x-x_{0}\right\|<r\right\}$.

Proof. Observe that if $x_{n}, y_{n} \in B_{r}\left(x_{0}\right)$, for all $n \geq 0$, then by Assumption 2, we have

$$
\begin{aligned}
x_{n}-y_{n-1} & \\
= & -R_{\alpha}\left(x_{0}\right)^{-1} A_{0}^{*}\left[F\left(y_{n-1}\right)-F\left(x_{n-1}\right)-A_{0}\left(y_{n-1}-x_{n-1}\right)\right] \\
= & R_{\alpha}\left(x_{0}\right)^{-1} A_{0}^{*} \\
& \times \int_{0}^{1}\left[F^{\prime}\left(y_{n-1}+t\left(x_{n-1}-y_{n-1}\right)\right)-A_{0}\right] \\
& \times\left(x_{n-1}-y_{n-1}\right) d t \\
= & R_{\alpha}\left(x_{0}\right)^{-1} A_{0}^{*} A_{0} \\
& \times \int_{0}^{1} \Phi\left(x_{0}, y_{n-1}+t\left(x_{n-1}-y_{n-1}\right), x_{n-1}-y_{n-1}\right) d t,
\end{aligned}
$$


and hence

$$
\left\|x_{n}-y_{n-1}\right\| \leq k_{0} r\left\|x_{n-1}-y_{n-1}\right\| .
$$

This proves (a).

Again observe that if $x_{n}, y_{n} \in B_{r}\left(x_{0}\right)$, for all $n \geq 0$,

$$
\begin{aligned}
y_{n}-x_{n}= & -R_{\alpha}\left(x_{0}\right)^{-1} A_{0}^{*} \\
& \times\left[\left(F\left(x_{n}\right)-F\left(y_{n-1}\right)\right)-A_{0}\left(x_{n}-y_{n-1}\right)\right] \\
= & -R_{\alpha}\left(x_{0}\right)^{-1} A_{0}^{*} \\
& \times \int_{0}^{1}\left[F^{\prime}\left(y_{n-1}+t\left(x_{n}-y_{n-1}\right)\right)-A_{0}\right] \\
& \times d t\left(x_{n}-y_{n-1}\right) \\
= & -R_{\alpha}\left(x_{0}\right)^{-1} A_{0}^{*} A_{0} \\
& \times \int_{0}^{1} \Phi\left(x_{0}, y_{n-1}+t\left(x_{n}-y_{n-1}\right), x_{n}-y_{n-1}\right) d t,
\end{aligned}
$$

and hence by Assumption 2 and (27), we have

$$
\left\|y_{n}-x_{n}\right\| \leq k_{0} r\left\|x_{n}-y_{n-1}\right\| \leq q^{2}\left\|x_{n-1}-y_{n-1}\right\| .
$$

This proves (b).

Thus, if $x_{n}, y_{n} \in B_{r}\left(x_{0}\right)$, for all $n \geq 0$, then (c) follows from (a) and (b). Now, we will prove using induction that $x_{n}, y_{n} \in$ $B_{r}\left(x_{0}\right)$, for all $n \geq 0$. Note that $x_{0}, y_{0} \in B_{r}\left(x_{0}\right)$, and hence by (27) and Remark 3,

$$
\begin{aligned}
\left\|x_{1}-x_{0}\right\| & \leq\left\|x_{1}-y_{0}\right\|+\left\|y_{0}-x_{0}\right\| \\
& \leq(1+q) e_{0} \\
& \leq \frac{\gamma_{\rho}}{1-q} \\
& \leq r
\end{aligned}
$$

that is, $x_{1} \in B_{r}\left(x_{0}\right)$, again by (29) and Remark 3 ,

$$
\begin{aligned}
\left\|y_{1}-x_{0}\right\| & \leq\left\|y_{1}-x_{1}\right\|+\left\|x_{1}-x_{0}\right\| \\
& \leq q^{2} e_{0}+(1+q) e_{0} \\
& \leq \frac{\gamma_{\rho}}{1-q} \\
& \leq r
\end{aligned}
$$

that is, $y_{1} \in B_{r}\left(x_{0}\right)$. Suppose that $x_{k}, y_{k} \in B_{r}\left(x_{0}\right)$ for some $k>1$. Then, since

$$
\left\|x_{k+1}-x_{0}\right\| \leq\left\|x_{k+1}-x_{k}\right\|+\left\|x_{k}-x_{k-1}\right\|+\cdots+\left\|x_{1}-x_{0}\right\|,
$$

we shall first find an estimate for $\left\|x_{k+1}-x_{k}\right\|$. Note that by (a), (b), and (c), we have

$$
\begin{aligned}
\left\|x_{k+1}-x_{k}\right\| & \leq\left\|x_{k+1}-y_{k}\right\|+\left\|y_{k}-x_{k}\right\| \\
& \leq(q+1)\left\|y_{k}-x_{k}\right\| \\
& \leq(1+q) q^{2 k} e_{0} .
\end{aligned}
$$

Therefore by (32) and Remark 3, we have

$$
\begin{aligned}
\left\|x_{k+1}-x_{0}\right\| & \leq(1+q)\left[q^{2 k}+q^{2(k-1)}+\cdots+1\right] e_{0} \\
& \leq(1+q) \frac{1-q^{2 k+1}}{1-q^{2}} e_{0} \\
& \leq \frac{\gamma_{\rho}}{1-q} \\
& \leq r,
\end{aligned}
$$

that is, $x_{k+1} \in B_{r}\left(x_{0}\right)$. So by induction $x_{n} \in B_{r}\left(x_{0}\right)$ for all $n \geq 0$. Again by (a), (b) and (34) we have

$$
\begin{aligned}
\left\|y_{k+1}-x_{0}\right\| & \leq\left\|y_{k+1}-x_{k+1}\right\|+\left\|x_{k+1}-x_{0}\right\| \\
& \leq q^{2 k+2} e_{0}+(1+q)\left[q^{2 k}+q^{2(k-1)}+\cdots+1\right] e_{0} \\
& \leq(1+q) \frac{1-q^{2 k+3}}{1-q^{2}} e_{0} \\
& \leq \frac{\gamma_{\rho}}{1-q} \\
& \leq r .
\end{aligned}
$$

Thus, $y_{k+1} \in B_{r}\left(x_{0}\right)$, and hence by induction, $y_{n} \in B_{r}\left(x_{0}\right)$ for all $n \geq 0$. This completes the proof of the theorem.

The main result of Section 2 is the following.

Theorem 5. Let $y_{n}$ and $x_{n}$ be as in (19) and (20), respectively, with $\delta \in\left(0, \delta_{0}\right]$, and assumptions of Theorem 4 hold. Then, $\left(x_{n}\right)$ is a Cauchy sequence in $B_{r}\left(x_{0}\right)$ and converges to $x_{\alpha}^{\delta} \epsilon$ $\overline{B_{r}\left(x_{0}\right)}$. Further, $A_{0}^{*}\left(F\left(x_{\alpha}^{\delta}\right)-y^{\delta}\right)+\alpha\left(x_{\alpha}^{\delta}-x_{0}\right)=0$, and

$$
\left\|x_{n}-x_{\alpha}^{\delta}\right\| \leq \frac{q^{2 n} \gamma_{\rho}}{(1-q)} .
$$

Proof. Using the relation (33), we obtain

$$
\begin{aligned}
\left\|x_{n+m}-x_{n}\right\| & \leq \sum_{i=0}^{i=m-1}\left\|x_{n+i+1}-x_{n+i}\right\| \\
& \leq \sum_{i=0}^{i=m-1}(1+q) q^{2(n+i)} e_{0} \\
& \leq(1+q) \frac{\left(q^{2 n}-q^{2 n+2 m}\right)}{1-q^{2}} e_{0} \\
& \leq \frac{q^{2 n}}{1-q} \gamma_{\rho} .
\end{aligned}
$$


Thus, $x_{n}$ is a Cauchy sequence in $B_{r}\left(x_{0}\right)$, and hence it converges, say to $x_{\alpha}^{\delta} \in \overline{B_{r}\left(x_{0}\right)}$. Observe that $\left\|x_{n+1}-y_{n}\right\| \leq$ $k_{0} r\left\|x_{n}-y_{n}\right\|$ and $k_{0} r=q<1$. Hence, $\left(y_{n}\right)$ also converges to $x_{\alpha}^{\delta}$.

Now, by $n \rightarrow \infty$ in (20), we obtain $x_{\alpha}^{\delta}=x_{0}-$ $\left(A_{0}^{*} A_{0}+\alpha I\right)^{-1} A_{0}^{*}\left[F\left(x_{\alpha}^{\delta}\right)-y^{\delta}-A_{0}\left(x_{\alpha}^{\delta}-x_{0}\right)\right]$, that is,

$$
A_{0}^{*}\left(F\left(x_{\alpha}^{\delta}\right)-y^{\delta}\right)+\alpha\left(x_{\alpha}^{\delta}-x_{0}\right)=0 .
$$

This completes the proof.

\section{Error Analysis}

The next assumption on source condition is based on a source function $\varphi$ and a property of the source function $\varphi$. We will be using this assumption to obtain an error estimate for $\left\|x_{\alpha}^{\delta}-\widehat{x}\right\|$.

Assumption 6. There exists a continuous, strictly monotonically increasing function $\varphi:(0, a] \rightarrow(0, \infty)$ with $a \geq$ $\left\|F^{\prime}\left(x_{0}\right)\right\|^{2}$ satisfying

(i) $\lim _{\lambda \rightarrow 0} \varphi(\lambda)=0$,

(ii) $\sup _{\lambda \geq 0}(\alpha \varphi(\lambda) /(\lambda+\alpha)) \leq \varphi(\alpha)$, for all $\lambda \in(0, a]$,

(iii) there exists $v \in X$ such that

$$
x_{0}-\widehat{x}=\varphi\left(A_{0}^{*} A_{0}\right) v \text {. }
$$

Theorem 7. Let $x_{\alpha}^{\delta}$ be as in (38). Then,

$$
\left\|x_{\alpha}^{\delta}-\widehat{x}\right\| \leq \frac{1}{1-q}\left(\frac{\delta}{\sqrt{\alpha}}+\varphi(\alpha)\right) .
$$

Proof. Let $M=\int_{0}^{1} F^{\prime}\left(\widehat{x}+t\left(x_{\alpha}^{\delta}-\widehat{x}\right)\right) d t$. Then,

$$
F\left(x_{\alpha}^{\delta}\right)-F(\widehat{x})=M\left(x_{\alpha}^{\delta}-\widehat{x}\right),
$$

and hence by (38), we have $\left(A_{0}^{*} M+\alpha I\right)\left(x_{\alpha}^{\delta}-\widehat{x}\right)=A_{0}^{*}\left(y^{\delta}-\right.$ $y)+\alpha\left(x_{0}-\widehat{x}\right)$. Thus,

$$
\begin{aligned}
x_{\alpha}^{\delta}-\widehat{x}= & \left(A_{0}^{*} A_{0}+\alpha I\right)^{-1} \\
& \times\left[A_{0}^{*}\left(y^{\delta}-y\right)+\alpha\left(x_{0}-\widehat{x}\right)+A_{0}^{*}\left(A_{0}-M\right)\left(x_{\alpha}^{\delta}-\widehat{x}\right)\right] \\
= & s_{1}+s_{2}+s_{3},
\end{aligned}
$$

where $s_{1}:=\left(A_{0}^{*} A_{0}+\alpha I\right)^{-1} A_{0}^{*}\left(y^{\delta}-y\right), s_{2}:=\left(A_{0}^{*} A_{0}+\right.$ $\alpha I)^{-1} \alpha\left(x_{0}-\widehat{x}\right)$, and $s_{3}:=\left(A_{0}^{*} A_{0}+\alpha I\right)^{-1} A_{0}^{*}\left(A_{0}-M\right)\left(x_{\alpha}^{\delta}-\widehat{x}\right)$. Note that

$$
\left\|s_{1}\right\| \leq \frac{\delta}{\sqrt{\alpha}}
$$

by Assumption 6

$$
\left\|s_{2}\right\| \leq \varphi(\alpha)
$$

and by Assumption 2

$$
\left\|s_{3}\right\| \leq k_{0} r\left\|x_{\alpha}^{\delta}-\hat{x}\right\| .
$$

The result now follows from (42), (43), (44), and (45). This completes the proof of the theorem.
3.1. Error Bounds under Source Conditions. Combining the estimates in Theorems 5 and 7, we obtain the following.

Theorem 8. Let $x_{n}^{\delta}$ be defined as in (20). If all assumptions of Theorems 5 and 7 are fulfilled, then

$$
\left\|x_{n}^{\delta}-\hat{x}\right\| \leq \frac{q^{2 n}}{1-q} \gamma_{\rho}+\frac{1}{1-q}\left(\frac{\delta}{\sqrt{\alpha}}+\varphi(\alpha)\right) .
$$

Further, if $n_{\delta}:=\min \left\{n: q^{2 n}<\delta / \sqrt{\alpha}\right\}$, then

$$
\left\|x_{n_{\delta}}^{\delta}-\widehat{x}\right\| \leq \widetilde{C}\left(\frac{\delta}{\sqrt{\alpha}}+\varphi(\alpha)\right) \text {, }
$$

where $\widetilde{C}:=(1 /(1-q))\left(\gamma_{\rho}+1\right)$.

3.2. A Priori Choice of the Parameter. Observe that the upper bound $(\delta / \sqrt{\alpha})+\varphi(\alpha)$ in Theorem 8 is of optimal order for the choice $\alpha:=\alpha_{\delta}$ which satisfies $\delta / \sqrt{\alpha_{\delta}}=\varphi(\alpha)$. Now, using the function $\psi(\lambda):=\lambda \sqrt{\varphi^{-1}(\lambda)}, 0<\lambda \leq a$, we have $\delta=\sqrt{\alpha} \varphi(\alpha)=\psi(\varphi(\alpha))$ so that $\alpha_{\delta}=\varphi^{-1}\left[\psi^{-1}(\delta)\right]$. Here, $\varphi^{-1}$ means the inverse function of $\varphi$.

Theorem 9. Suppose that all assumptions of Theorems 5 and 7 are fulfilled. For $\delta>0$, let $\alpha_{\delta}=\varphi^{-1}\left[\psi^{-1}(\delta)\right]$, and let $n_{\delta}$ be as in Theorem 8 with $\alpha=\alpha_{\delta}$. Then,

$$
\left\|x_{n_{\delta}}^{\delta}-\hat{x}\right\|=O\left(\psi^{-1}(\delta)\right)
$$

3.3. Adaptive Choice of the Parameter. In the balancing principle considered by Pereverzev and Schock in [15], the regularization parameter $\alpha=\alpha_{i}$ is selected from some finite set

$$
D_{N}:=\left\{\alpha_{i}: 0<\alpha_{0}<\alpha_{1}<\cdots<\alpha_{N}\right\} .
$$

Let

$$
n_{i}=\min \left\{n: q^{2 n} \leq \frac{\delta}{\sqrt{\alpha_{i}}}\right\},
$$

and let $x_{n_{i}, \alpha_{i}}^{\delta}:=x_{n_{i}}^{\delta}$, where $x_{n_{i}}^{\delta}$ is defined as in (20) with $\alpha=\alpha_{i}$ and $n+1=n_{i}$. Then, from Theorem 8 , we have

$$
\left\|x_{n_{i}, \alpha_{i}}^{\delta}-\widehat{x}\right\| \leq \widetilde{C}\left(\frac{\delta}{\sqrt{\alpha_{i}}}+\varphi\left(\alpha_{i}\right)\right), \quad \forall i=1,2, \ldots, N .
$$

Precisely, we choose the regularization parameter $\alpha=\alpha_{k}$ from the set $D_{N}$ defined by

$$
D_{N}:=\left\{\alpha_{i}=\mu^{i} \alpha_{0}, i=1,2, \ldots, N\right\},
$$

where $\mu>1$.

To obtain a conclusion from this parameter choice, we consider all possible functions $\varphi$ satisfying Assumptions 2 and $\varphi\left(\alpha_{i}\right) \leq \delta / \sqrt{\alpha_{i}}$. Any of such functions is called admissible for $\widehat{x}$, and it can be used as a measure for the convergence of $x_{\alpha}^{\delta} \rightarrow \widehat{x}$ (see [16]).

The main result of Section 3 is the following. 


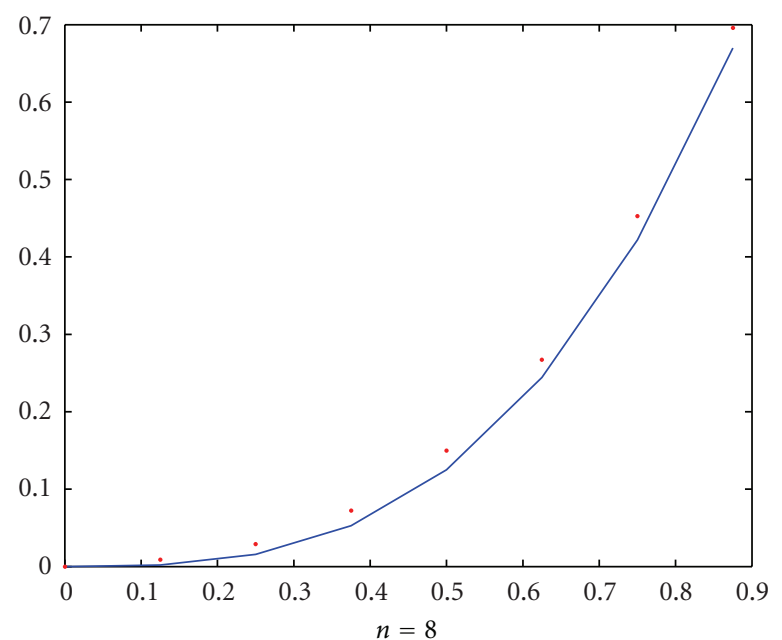

- Exact solution

Apprx. solution

(a)

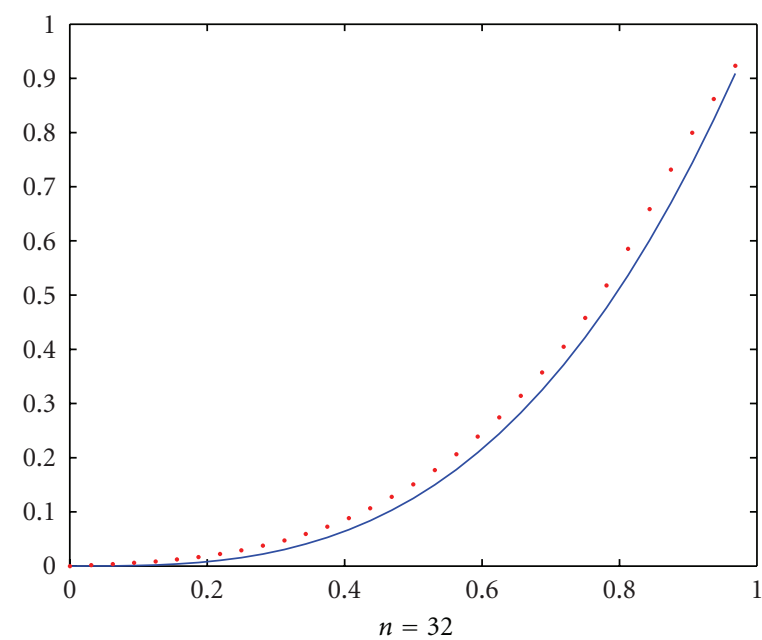

- Exact solution
Apprx. solution

$n=32$

(c)

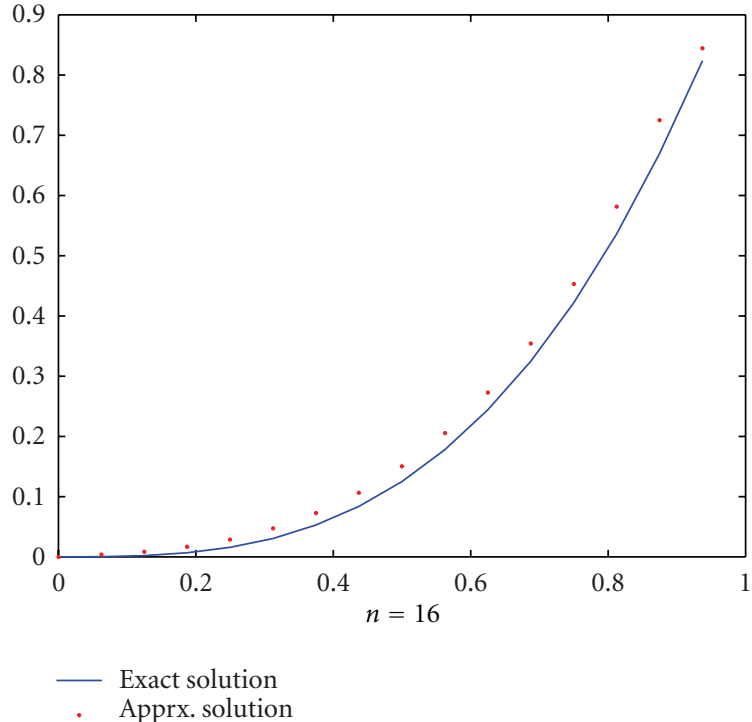

(b)

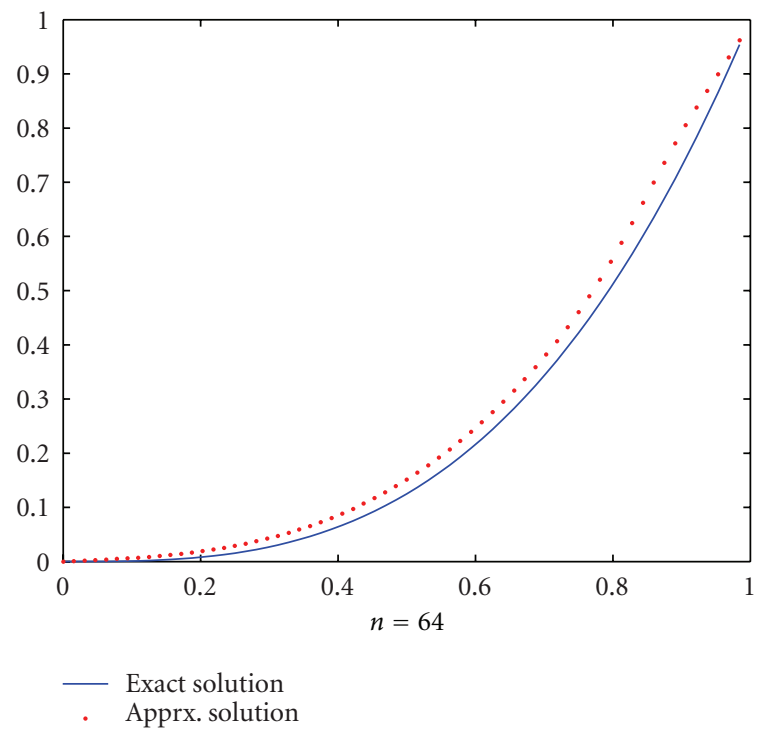

(d)

FIgURE 1: Curves of the exact and approximate solutions.

Theorem 10. Assume that there exists $i \in\{0,1, \ldots, N\}$ such that $\varphi\left(\alpha_{i}\right) \leq \delta / \sqrt{\alpha_{i}}$. Let the assumptions of Theorems 5 and 7 be fulfilled, and let

$$
\begin{gathered}
l:=\max \left\{i: \varphi\left(\alpha_{i}\right) \leq \frac{\delta}{\sqrt{\alpha_{i}}}\right\}<N, \\
k=\max \left\{i: \forall j=1,2, \ldots, i ;\left\|x_{n_{i}, \alpha_{i}}^{\delta}-x_{n_{j}, \alpha_{j}}^{\delta}\right\| \leq 4 \widetilde{C} \frac{\delta}{\sqrt{\alpha_{j}}}\right\},
\end{gathered}
$$

where $\widetilde{C}$ is as in Theorem 8 . Then, $l \leq k$ and

$$
\left\|x_{n_{k}, \alpha_{k}}^{\delta}-\widehat{x}\right\| \leq 6 \widetilde{C} \mu \psi^{-1}(\delta) .
$$

Proof. The proof is analogous to the proof of Theorem $4.4 \mathrm{in}$ [13] and is omitted therefore here.

\section{Implementation of the Method}

Finally, the balancing algorithm associated with the choice of the parameter specified in Theorem 10 involves the following steps.

(i) Choose $\alpha_{0}>0$ such that $\delta_{0}<\sqrt{\alpha_{0}} / 4 k_{0}$ and $\mu>1$.

(ii) Choose $N$ big enough but not too large and $\alpha_{i}:=\mu^{i} \alpha_{0}$, $i=0,1,2, \ldots, N$.

(iii) Choose $\rho \leq\left(\sqrt{(3 / 2)-\left(2 k_{0} \delta_{0} / \sqrt{\alpha_{0}}\right)}-1\right) / k_{0}$. 


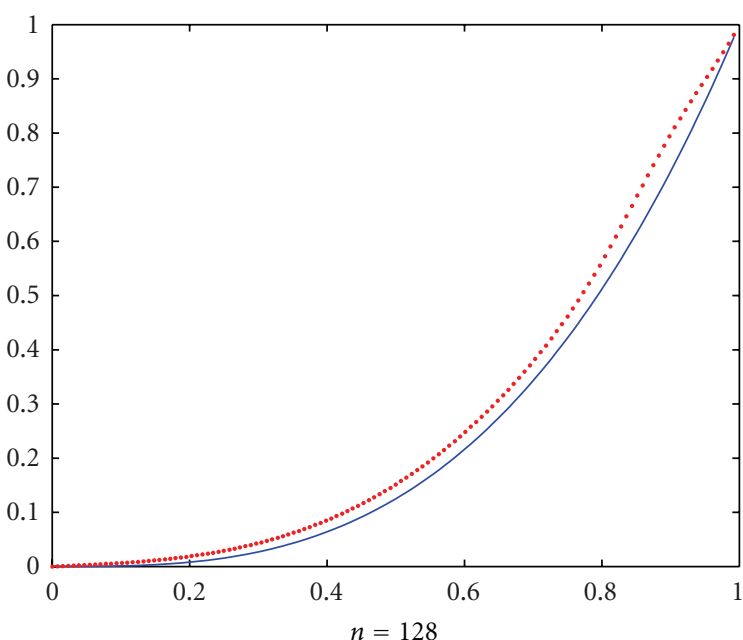

- Exact solution Apprx. solution

(a)

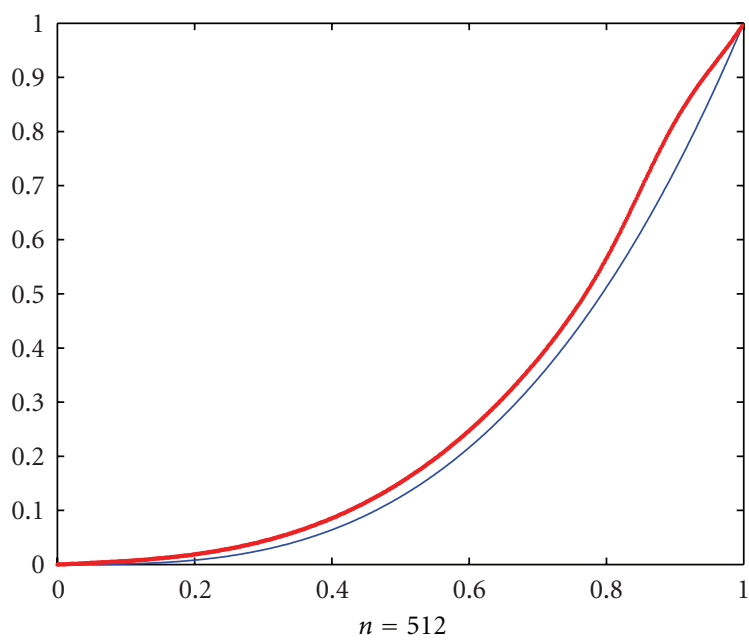

Exact solution Apprx. solution

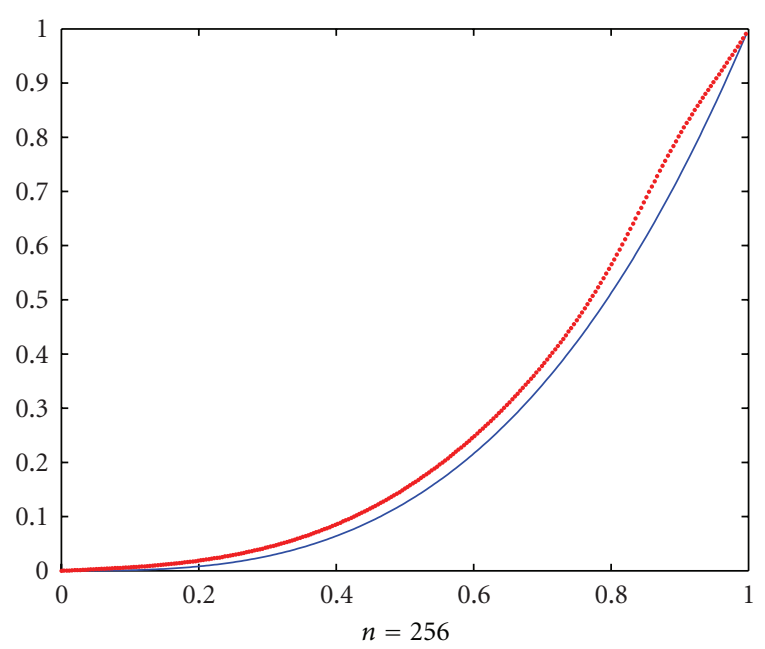

- Exact solution Apprx. solution

(b)

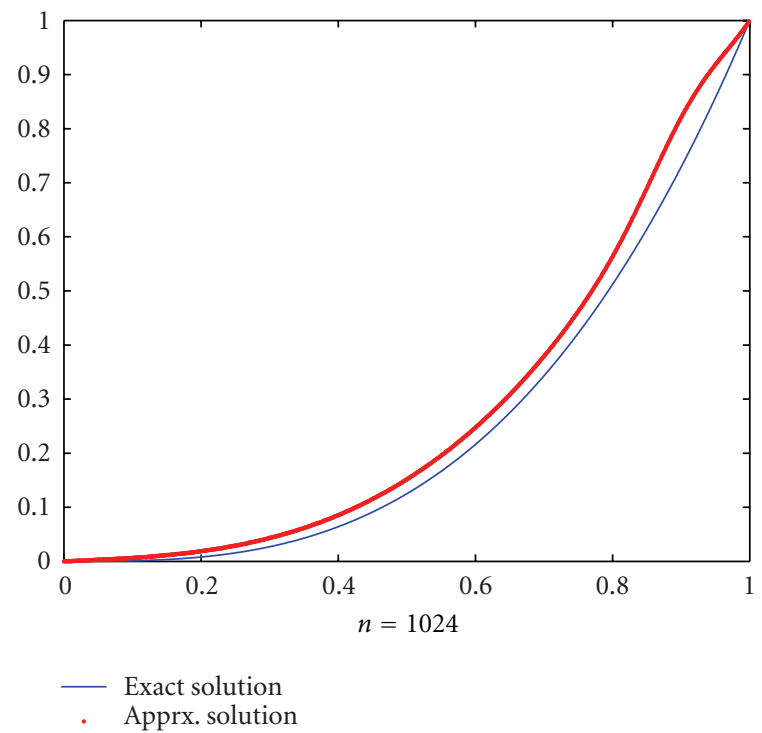

(d)

(c)

Figure 2: Curves of the exact and approximate solutions.

TABLE 1: Iterations and corresponding error estimates.

\begin{tabular}{llccccc}
\hline$n$ & $k$ & $n_{k}$ & $\delta+\varepsilon_{n}$ & $\alpha_{k}$ & $\left\|x_{n_{k}, \alpha_{k}}^{\delta}-\widehat{x}\right\|$ & $\left\|x_{n_{k}, \alpha_{k}}-\widehat{x}\right\| /\left(\delta+\varepsilon_{n}\right)^{1 / 2}$ \\
\hline 8 & 2 & 6 & 0.1016 & 0.8483 & 0.2622 & 0.8228 \\
16 & 2 & 6 & 0.1004 & 0.8385 & 0.1958 & 0.6179 \\
32 & 2 & 6 & 0.1001 & 0.8360 & 0.1438 & 0.4545 \\
64 & 2 & 6 & 0.1000 & 0.8354 & 0.1056 & 0.3338 \\
128 & 2 & 6 & 0.1000 & 0.8353 & 0.0661 & 0.2495 \\
256 & 2 & 6 & 0.1000 & 0.8352 & 0.0522 & 0.1948 \\
512 & 2 & 6 & 0.1000 & 0.8352 & 0.0459 & 0.1652 \\
1024 & 2 & 6 & 0.1000 & 0.8352 & & 0.1450 \\
\hline
\end{tabular}




\subsection{Algorithm}

(1) Set $i=0$.

(2) Choose $n_{i}=\min \left\{n: q^{2 n} \leq \delta / \sqrt{\alpha_{i}}\right\}$.

(3) Solve $x_{n_{i}, \alpha_{i}}^{\delta}=x_{n_{i}}^{\delta}$ by using the iteration in (19) and (20) with $n=n_{i}$ and $\alpha=\alpha_{i}$.

(4) If $\left\|x_{\alpha_{i}}^{\delta}-x_{\alpha_{j}}^{\delta}\right\|>4 \widetilde{C}\left(\delta / \sqrt{\alpha_{j}}\right), j<i$, then take $k=i-1$ and return $x_{\alpha_{k}}^{\delta}$.

(5) Else set $i=i+1$, and return to step (2).

\section{Numerical Example}

We apply the algorithm by choosing a sequence of finite dimensional subspace $\left(V_{n}\right)$ of $X$ with $\operatorname{dim} V_{n}=n+1$. Precisely, we choose $V_{n}$ as the linear span of $\left\{v_{1}, v_{2}, \ldots, v_{n+1}\right\}$, where $v_{i}$, $i=1,2, \ldots, n+1$ are the linear splines in a uniform grid of $n+1$ points in $[0,1]$.

We consider the same example of nonlinear integral operator as in [17, Section 4.3]. Let $F: D(F) \subseteq L^{2}(0,1) \rightarrow$ $L^{2}(0,1)$ be defined by

$$
F(u):=\int_{0}^{1} k(t, s) u^{3}(s) d s,
$$

where

$$
k(t, s)= \begin{cases}(1-t) s, & 0 \leq s \leq t \leq 1 \\ (1-s) t, & 0 \leq t \leq s \leq 1\end{cases}
$$

The Fréchet derivative of $F$ is given by

$$
F^{\prime}(u) w=3 \int_{0}^{1} k(t, s)(u(s))^{2} w(s) d s .
$$

Note that for $u, v>0$,

$$
\begin{aligned}
\left(F^{\prime}(v)-F^{\prime}(u)\right) w= & \left(3 \int_{0}^{1} k(t, s)(u(s))^{2} d s\right) \\
& \times\left[\frac{\int_{0}^{1} k(t, s)\left((v(s))^{2}-(u(s))^{2}\right) w(s) d s}{\int_{0}^{1} k(t, s)(u(s))^{2} d s}\right] \\
:= & F^{\prime}(u) \Phi(v, u, w),
\end{aligned}
$$

where $\Phi(v, u, w)=\left(\int_{0}^{1} k(t, s)\left((v(s))^{2}-(u(s))^{2}\right) w(s) d s\right) /$ $\left(\int_{0}^{1} k(t, s)(u(s))^{2} d s\right)$.

Observe that

$$
\begin{aligned}
\Phi(v, u, w) & =\frac{\int_{0}^{1} k(t, s)\left((v(s))^{2}-\left((u(s))^{2}\right) w(s) d s\right.}{\int_{0}^{1} k(t, s)(u(s))^{2} d s} \\
& =\frac{\int_{0}^{1} k(t, s)(u(s)+v(s))(v(s)-u(s)) w(s) d s}{\int_{0}^{1} k(t, s)(u(s))^{2} d s} .
\end{aligned}
$$

So, Assumption 2 is satisfied with $k_{0} \geq \|\left(\int_{0}^{1} k(t, s)(u(s)+\right.$ $v(s)) d s) /\left(\int_{0}^{1} k(t, s)(u(s))^{2} d s\right) \|$

In our computation, we take $y(t)=\left(t-t^{11}\right) / 110$ and $y^{\delta}=$ $y+\delta$. Then, the exact solution

$$
\widehat{x}(t)=t^{3} \text {. }
$$

We use

$$
x_{0}(t)=t^{3}+\frac{3}{56}\left(t-t^{8}\right)
$$

as our initial guess, so that the function $x_{0}-\widehat{x}$ satisfies the source condition

$$
x_{0}-\widehat{x}=\varphi\left(F^{\prime}\left(x_{0}\right)\right)\left(\frac{\widehat{x}}{x_{0}}\right)^{2},
$$

where $\varphi(\lambda)=\lambda$.

Observe that while performing numerical computation on finite dimensional subspace $\left(V_{n}\right)$ of $X$, one has to consider the operator $P_{n} F^{\prime}(\cdot) P_{n}$ instead of $F^{\prime}(\cdot)$, where $P_{n}$ is the orthogonal projection on to $V_{n}$. Thus, incurs an additional error $\left\|P_{n} F^{\prime}(\cdot) P_{n}-F^{\prime}(\cdot)\right\|=O\left(\left\|F^{\prime}(\cdot)\left(I-P_{n}\right)\right\|\right)$.

Let $\left\|F^{\prime}(\cdot)\left(I-P_{n}\right)\right\| \leq \varepsilon_{n}$. For the operator $F^{\prime}(\cdot)$ defined in (57), $\varepsilon_{n}=O\left(n^{-2}\right)$ (cf. [18]). Thus, we expect to obtain the rate of convergence $O\left(\left(\delta+n^{-2}\right)^{1 / 2}\right)$.

We choose $\alpha_{0}=1.7 \times\left(\delta+\varepsilon_{n}\right), \mu=1.7, k_{0}=1$, and $q=$ 0.71 . The results of the computation are presented in Table 1. The plots of the exact solution and the approximate solution obtained are given in Figures 1 and 2.

\section{Conclusion}

In this paper, we considered a modified Gauss-Newton method for approximately solving the nonlinear ill-posed operator equation $F(x)=y$, where $F: D(F) \subseteq X \rightarrow Y$ is a nonlinear operator between the Hilbert spaces $X$ and $Y$. The same method was considered in [13] by the author, but the analysis in [13] was based on a suitably constructed majorizing sequence. In this paper, we analyze the sequence by considering its even and odd terms separately. The analysis in this paper is easier than that of [13]. We use the adaptive method considered by Pereverzev and Schock in [15] for choosing the regularization parameter. The optimality of this method is proved under a general source condition. Finally, a numerical example of nonlinear integral equation shows the performance of this method.

\section{References}

[1] J. Hadamard, Lectures on Cauchy's Problem in Linear Partial Differential Equations, Dover Publications, New York, NY, USA, 1952.

[2] H. W. Engl, K. Kunisch, and A. Neubauer, Regularization of Inverse Problems, Kluwer Academic, Dordrecht, The Netherlands, 1996.

[3] H. W. Engl, K. Kunisch, and A. Neubauer, "Convergence rates for Tikhonov regularisation of non-linear ill-posed problems," Inverse Problems, vol. 5, no. 4, pp. 523-540, 1989. 
[4] A. B. Bakushinskii, "The problem of the convergence of the iteratively regularized Gauss-Newton method," Computational Mathematics and Mathematical Physics, vol. 32, no. 9, pp. 13531359, 1992.

[5] A. B. Bakushinskii, "Iterative methods without saturation for solving degenerate nonlinear operator equations," Doklady Akademii Nauk, vol. 344, pp. 7-8, 1995.

[6] B. Blaschke, A. Neubauer, and O. Scherzer, "On convergence rates for the iteratively regularized Gauss-Newton method," IMA Journal of Numerical Analysis, vol. 17, no. 3, pp. 421-436, 1997.

[7] B. Kaltenbacher, "A note on logarithmic convergence rates for nonlinear Tikhonov regularization," Journal of Inverse and IllPosed Problems, vol. 16, no. 1, pp. 79-88, 2008.

[8] P. A. Mahale and M. T. Nair, "A simplified generalized GaussNewton method for nonlinear ill-posed problems," Mathematics of Computation, vol. 78, no. 265, pp. 171-184, 2009.

[9] T. Hohage, "Logarithmic convergence rates of the iteratively regularized Gauss-Newton method for an inverse potential and an inverse scattering problem," Inverse Problems, vol. 13, no. 5, pp. 1279-1299, 1997.

[10] T. Hohage, "Regularization of exponentially ill-posed problems," Numerical Functional Analysis and Optimization, vol. 21, no. 3, pp. 439-464, 2000.

[11] S. Langer and T. Hohage, "Convergence analysis of an inexact iteratively regularized Gauss-Newton method under general source conditions," Journal of Inverse and Ill-Posed Problems, vol. 15, no. 3, pp. 311-327, 2007.

[12] A. Bakushinsky and A. Smirnova, "On application of generalized discrepancy principle to iterative methods for nonlinear illposed problems," Numerical Functional Analysis and Optimization, vol. 26, no. 1, pp. 35-48, 2005.

[13] S. George, "On convergence of regularized modified Newton's method for nonlinear ill-posed problems," Journal of Inverse and Ill-Posed Problems, vol. 18, no. 2, pp. 133-146, 2010.

[14] I. K. Argyros, Convergence and Applications of Newton-Type Iterations, Springer, New York, NY, USA, 2008.

[15] S. Pereverzev and E. Schock, "On the adaptive selection of the parameter in regularization of ill-posed problems," SIAM Journal on Numerical Analysis, vol. 43, no. 5, pp. 2060-2076, 2005.

[16] S. Lu and S. V. Pereverzev, "Sparsity reconstruction by the standard Tikhonov method," RICAM-Report 2008-17, 2008.

[17] E. V. Semenova, "Lavrentiev regularization and balancing principle for solving ill-posed problems with monotone operators," Computational Methods in Applied Mathematics, vol. 4, no. 4, pp. 444-454, 2010.

[18] C. W. Groetsch, J. T. King, and D. Murio, "Asymptotic analysis of a finite element method for Fredholm equations of the first kind," in Treatment of Integral Equations by Numerical Methods, C. T. H. Baker and G. F. Miller, Eds., pp. 1-11, Academic Press, London, UK, 1982. 


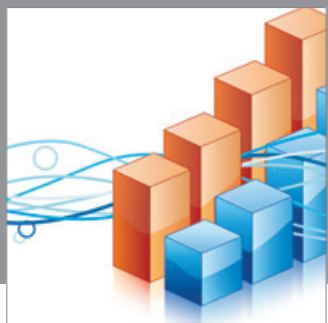

Advances in

Operations Research

mansans

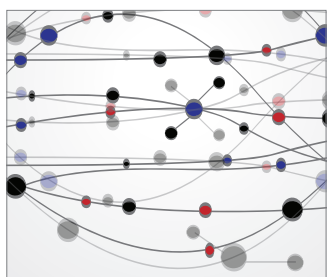

The Scientific World Journal
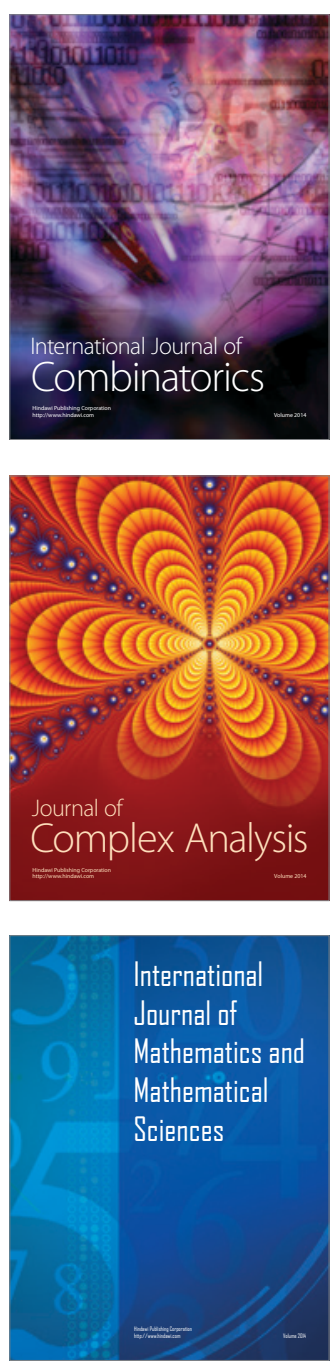
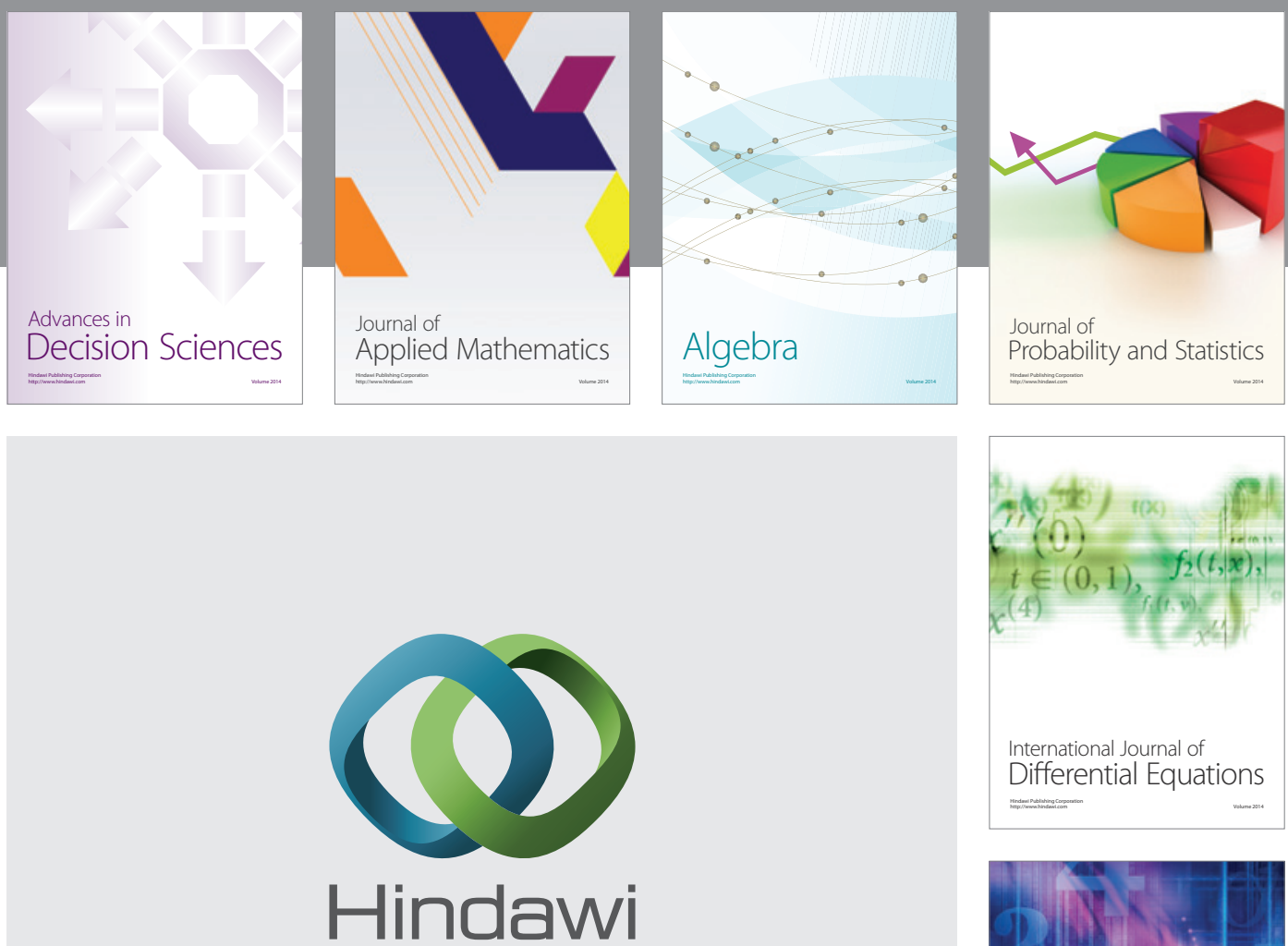

Submit your manuscripts at http://www.hindawi.com
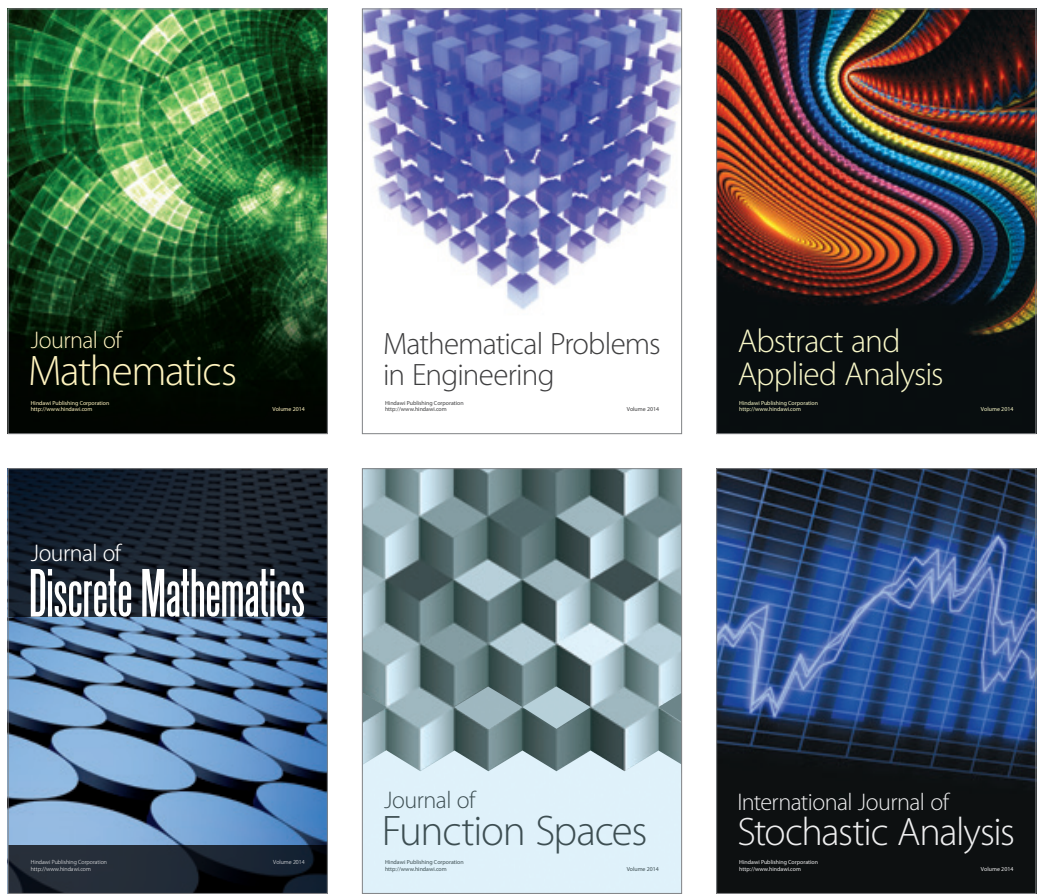

Journal of

Function Spaces

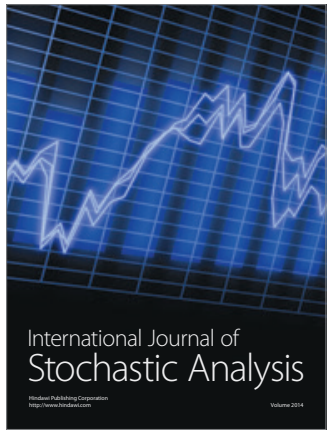

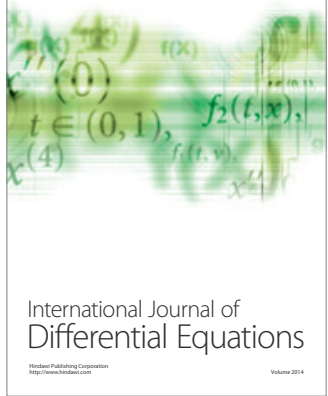
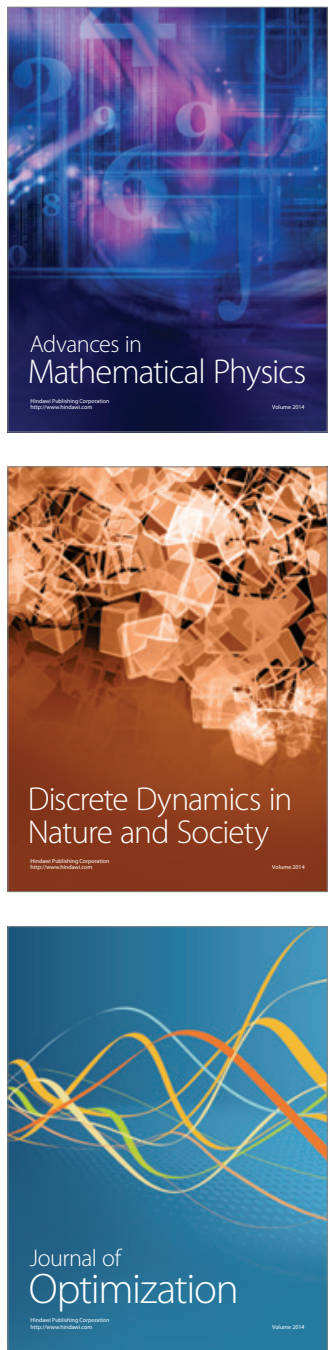